
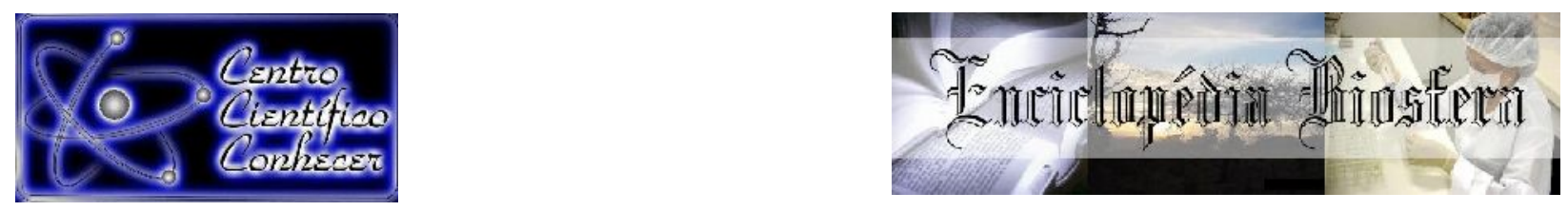

\title{
SISTEMA PENITENCIÁRIO E SAÚDE: AVALIAÇÃO DAS CONDIÇÕES BUCAIS DE DETENTOS DA REGIÃO METROPOLITANA DE SALVADOR, BA
}

\author{
Sandy Guimarães Cruz Damasceno ${ }^{1}$, Roberto Carlos Carvalho Cerqueira ${ }^{1}$, \\ João Rubens Teixeira de Castro Silva ${ }^{2}$, Kaliane Rocha Soledade ${ }^{3}$, \\ Larissa Rolim Borges-Paluch ${ }^{3}$ \\ ${ }^{1}$ Graduado em Odontologia pela Faculdade Maria Milza (FAMAM), Governador \\ Mangabeira, BA, Brasil. \\ ${ }^{2}$ Mestrando em Desenvolvimento Regional e Meio Ambiente da FAMAM, \\ Governador Mangabeira, BA. \\ ${ }^{3}$ Professora Doutora do Mestrado em Desenvolvimento Regional e Meio Ambiente e \\ Biotecnologia da FAMAM, Gov. Mangabeira, BA. \\ E-mail: larissapaluch@gmail.com
}

Recebido em: 15/11/2020 - Aprovado em: 15/12/2020 - Publicado em: 30/12/2020

DOI: 10.18677/EnciBio_2020D36

\begin{abstract}
RESUMO
As dificuldades do sistema penitenciário refletem diretamente na saúde dos encarcerados, inclusive em relação a assistência odontológica de forma integral e efetiva. O estudo teve como objetivo caracterizar os indivíduos quanto as variáveis sociodemográficas e avaliar as condições de saúde bucal de internos de um sistema prisional de Lauro de Freitas, BA. Trata-se de um estudo epidemiológico descritivo, de delineamento transversal e abordagem quantitativa. A coleta de dados foi realizada entre abril e maio/2018 por meio da análise dos prontuários odontológicos de homens que se encontram em cárcere. A amostra foi composta por 239 prontuários e foi observado que, em relação às características sociodemográficas, $57,3 \%$ dos presidiários estão na faixa etária entre 20 e 30 anos; $70,3 \%$ se autodeclararam faioderma (pardo) e 57,7\% estudaram até o ensino fundamental. A cárie dentária foi a doença mais identificada nos odontogramas, acometendo $24,5 \%$ dos dentes da população. A mediana do índice de dentes cariados, perdidos e obturados (CPO-D) médio foi classificada como moderado, correspondendo a 13,1 e com o componente 'dentes cariados' sendo o problema mais prevalente. Tais achados evidenciaram a necessidade de orientações adequadas para a realização da higienização bucal por parte dos odontólogos, visando à prevenção da doença cárie e novas perdas dentárias. Além disso, é necessário a reabilitação e tratamento dos problemas odontológicos existentes, inclusive decorrentes da negligência anterior ao confinamento, visando proporcionar melhor qualidade de vida a este grupo de indivíduos.
\end{abstract}

PALAVRAS-CHAVE: inquéritos de saúde bucal; prisões; saúde das minorias étnicas 


\title{
PENITENTIARY AND HEALTH SYSTEM: EVALUATION OF THE ORAL CONDITIONS OF PRISONER IN METROPOLITAN REGION OF SALVADOR, BA
}

\begin{abstract}
The difficulties of the prison system directly reflect on the health of prisoners, including in relation to dental care in a comprehensive and effective way. The study aimed to characterize the individuals regarding sociodemographic variables and to evaluate the oral health conditions of inmates of a prison system in Lauro de Freitas, BA. This is a descriptive epidemiological study, with a cross-sectional design and a quantitative approach. Data collection was performed between April and May/2018 through the analysis of dental records of men who are in prison. The sample consisted of 239 medical records and it was observed that, in relation to sociodemographic characteristics, $57.3 \%$ of the inmates are between 20 and 30 years old; $70.3 \%$ declared themselves faioderma (brown) and $57.7 \%$ studied until elementary school. Dental caries was the most identified disease in odontograms, affecting $24.5 \%$ of the teeth of the population. The median index of decayed, ordered and filling teeth (DMT) was classified as moderate, corresponding to 13.1 and with the component 'decayed teeth' being the most prevalent problem. These findings showed the need for adequate guidance for oral hygiene by dentists, aiming at the prevention of caries disease and new tooth loss. In addition, it is necessary to rehabilitated and treat existing dental problems, including due to pre-confinement neglect, in order to provide better quality of life and this group of individuals.
\end{abstract}

KEYWORDS: prisons. health of ethnic minorities. oral health surveys.

\section{INTRODUÇÃO}

Segundo dados do Levantamento Nacional de Informações Penitenciárias, o Brasil possui uma população prisional de 773.151 pessoas privadas de liberdade em todos os regimes, sendo observado um crescimento da população carcerária de 2017 para 2018 de 2,97\% e no último semestre de 2018 para o primeiro de 2019 de 3,89\% (INFOPEN, 2020).

Apesar da imensa quantidade de detentos, o sistema prisional brasileiro vivencia uma triste realidade em virtude da deficiência estrutural, superlotação, ausência de apoio psicológico, falta de saneamento básico e de investimentos. Este cenário contribui para o desenvolvimento e agravo de diversas enfermidades, afetando diretamente a saúde física e mental dos apenados e das pessoas que esses indivíduos mantém contato, direto ou indireto (SILVA, 2017).

A saúde é resultante de uma soma de fatores, onde condições legais, sociais, demográficas, comportamentais, econômicas, entre outras, influenciam de forma direta no bem estar do indivíduo, incluindo o poder de exercer sua liberdade. Neste cenário, a disponibilização de condições benéficas de vida para os detentos é dever do Estado. Para tanto, torna-se necessária a prestação da integralidade assistencial à saúde, uma vez que condições degradantes podem interferir negativamente na saúde desses cidadãos. Apesar de a saúde ser um direito constitucionalmente garantido, a realidade do sistema carcerário está distante do que está previsto, ou seja, não há uma assistência integral e efetiva (ALVES et al., 2017).

Além disso, outros obstáculos surgem no sistema carcerário como o ambiente infenso e amedrontador que afugenta profissionais aptos ao cargo. Assim, as práticas de saúde se tornam inexecutáveis, agravando ainda mais a situação dos encarcerados. Salienta-se também que outras dificuldades estão presentes em 
diversas instituições, como a falta de estrutura e segurança necessária para execução do trabalho (TETZNER et al., 2012).

No Brasil, até o ano de 2003, não havia uma política específica de saúde voltada para a população encarcerada. Visando aprovisionar essa necessidade, o governo, por meio do Ministério da Justiça e da Saúde, criou a portaria interministerial $n^{\circ}$ 1.777, de 9 de setembro de 2003, instituindo o Plano Nacional de Saúde no Sistema Penitenciário (PNSSP). O PNSSP incluiu a população penitenciária no Sistema Único de Saúde (SUS) e realizou diversas reformas no sistema penitenciário, inserindo ambulatórios visando atender as necessidades em vários níveis dessa população, com objetivo de garantir ações integrais de saúde que visem a assistência, bem como as ações de promoção e prevenção de saúde dessa população (FREITAS et al., 2016).

Em relação à redução dos agravos de saúde bucal da população carcerária pelo PNSSP, foram realizadas ações de promoção, prevenção e recuperação das funções mastigatórias, capacitando essa população quanto ao autocuidado com a saúde bucal, prevenção e detecção precoce do câncer de boca, doenças sistêmicas e virais com manifestações bucais. Estas ações são de fundamental importância levando-se em consideração as limitações e vulnerabilidades dessa população (TETZNER et al., 2012).

Dentre os principais problemas bucais dessa população observados em estudos do Ministério da Saúde, os mais frequentes são cárie, periodontite e mau hálito, devido a insuficiente ou inexistente higiene bucal. $\mathrm{O}$ cidadão, mesmo em regime de detenção tem o direito à saúde, sendo de responsabilidade da instituição proporcionar ao mesmo esse direito (BRASIL, 2010). Muitos dos problemas provavelmente ocorrem em função da negligência com cuidados anteriormente à prisão, bem como pelas dificuldades de acesso ou escassez de serviços odontológicos prestados pelo sistema penitenciário, o qual vivencia cotidianamente a grande demanda de serviços odontológicos, na maioria das vezes, em caráter de urgência. Por isso, visando a minimização, quando a instituição carcerária não disponibiliza os serviços necessários, os mesmos devem ser realizados em outros locais. Porém esta assistência deve ser realizada com segurança, devido à condição peculiar desses indivíduos, ou seja, todos os procedimentos devem ser realizados mediante a autorização dos órgãos jurídicos, e supervisão da instituição, garantindo que todos os direitos do detento sejam cumpridos (SILVA et al., 2016; SOARES FILHO; BUENO, 2016).

Com base nessas informações e buscando conhecer os principais problemas que acometem a cavidade bucal de indivíduos do sistema prisional, visando contribuir para a avaliação das ações de prevenção desses agravos e subsidiando o planejamento e gestão de políticas e ações voltadas à melhoria da saúde bucal dessa população, esta pesquisa teve como objetivo caracterizar os indivíduos quanto as variáveis sociodemográficas e avaliar as condições de saúde bucal (em relação ao número de dentes cariados, perdidos e obturados - CPO-D) de internos de um sistema prisional de Lauro de Freitas, BA.

\section{MATERIAIS E MÉTODOS}

Tratou-se de um estudo epidemiológico descritivo, de delineamento transversal e abordagem quantitativa. Sendo realizado em um Conjunto Penal no município de Lauro de Freitas, localizado na Região Metropolitana de Salvador, BA. A Instituição recebe homens infratores que cumprem pena em regime semiaberto, e recebe provisoriamente detentos em outros regimes. A mesma presta serviços 
odontológicos para os internos, atendendo as áreas de dentística, periodontia e cirurgia, executadas por cirurgião-dentista credenciado pela Instituição.

As informações de interesse da pesquisa foram coletadas de todos os prontuários odontológicos disponíveis na Instituição Carcerária. Os critérios de inclusão aplicados foram: ser detento da instituição durante o período da coleta de dados e ter se submetido a, pelo menos, um tratamento odontológico na instituição prisional. Os critérios de exclusão foram: o detento estar em caráter provisório na instituição e grafia ilegível no prontuário.

As coletas foram realizadas entre abril e maio/2018 sendo anotados em fichas específicas os dados relativos ao perfil sociodemográfico e levantamento de informações da condição bucal referente ao número do CPO-D da população. Após a obtenção dos dados, foi utilizado o programa Microsoft Office Excel, onde as informações obtidas dos prontuários foram organizadas de maneira sistemática e apresentadas por meio de tabelas e gráficos. A organização dos dados visou traçar o perfil sociodemográfico dos pacientes e calcular o índice CPO-D dividindo-se o valor encontrado pelo número de dentes analisados na pesquisa. A expressão destas informações ocorreu a partir da obtenção das frequências absolutas e relativas, além do cálculo de média aritmética simples.

O presente projeto possui aprovação do Comitê de Ética em Pesquisa da FAMAM, parecer 2.548.328, CAAE 83720017.0.0000.5025. E atentou para a fidelidade da pesquisa no que diz respeito a todos os princípios éticos contidos na Resolução $n^{\circ}$ 466/12 do Conselho Nacional de Saúde, assegurando confiabilidade, privacidade, anonimato e sigilo da identidade dos sujeitos da pesquisa e, ao mesmo tempo, garantindo imparcialidade, impessoalidade e objetividade na condução do estudo e na redação dos relatórios técnicos (BRASIL, 2012).

\section{RESULTADOS E DISCUSSÃO}

Foram analisados 411 prontuários odontológicos, dos quais depois de aplicados os critérios de inclusão e exclusão resultaram em uma amostra de 239 prontuários. Foi verificado que a idade média dos presidiários foi de 42,5 anos, com a mínima de 20 anos e a máxima de 65 anos. A distribuição dos apenados, segundo a faixa etária, revelou que a maioria tinha entre 20 e 30 anos (57,3\%); quanto à escolaridade, $138(57,7 \%)$ cursaram o ensino fundamental e de acordo a classificação cor/raça/etnia 168 (70,3\%) se autodeclararam faioderma ${ }^{1}$ (Tabela 1).

TABELA 1 - Frequências absoluta $(n)$ e relativa (\%) referentes aos dados sociodemográficos dos pacientes da unidade prisional de Lauro de Freitas, BA.

\begin{tabular}{|l|r|}
\hline Cor/Raça/Etnia & $\mathbf{n ~ ( \% )}$ \\
\hline Melanoderma & $53(22,2)$ \\
\hline Faioderma & $168(70,3)$ \\
\hline Leucoderma & $17(7,1)$ \\
\hline Eritroderma & $01(0,4)$ \\
\hline Faixa etária & $\mathbf{n ( \% )}$ \\
\hline $20-30$ & $137(57,3)$ \\
\hline $31-40$ & $68(28,5)$ \\
\hline $41-50$ & $26(10,9)$ \\
\hline
\end{tabular}

\footnotetext{
${ }^{1}$ A nomenclatura adotada para a identificação da raça/cor da pele na área médico-legal foi introduzida por Roquette-Pinto (1933), cujas denominações foram adaptadas do grego: melanoderma (melanodermos) para pretos; leucoderma (leucodermos) para brancos; faioderma (phaiodermos) para pardos e eritroderma para indígenas.
} 


\begin{tabular}{|l|r|}
\hline $51-65$ & $08(3,3)$ \\
\hline Escolaridade & $\mathbf{n}(\%)$ \\
\hline Analfabeto & $11(4,6)$ \\
\hline Fundamental & $138(57,7)$ \\
\hline Médio & $90(37,7)$ \\
\hline
\end{tabular}

Fonte: autores (2018).

Ao analisar estudos realizados em outras instituições carcerárias é possível identificar que, semelhante ao encontrado nesta pesquisa, há predominância de jovens entre os encarcerados. Siqueira et al. (2020) em relação a características sociodemográficas de encarcerados em uma cadeia pública do Estado do Pará, sendo observado que o maior número de detentos estava na faixa etária de 18 a 34 anos $(73,7 \%)$ e $75,96 \%$ com o grau de escolaridade até o ensino fundamental.

Dados do Cadastro Nacional de Presos também demonstram perfil semelhante em relação à população carcerária do País, em que 30,52\% estão na faixa etária entre 18 e 24 anos e $23,39 \%$ entre 25 e 29 anos de idade, constatando que mais da metade da população carcerária registrada tem até 29 anos. Quanto à cor/raça/etnia das pessoas privadas de liberdade no País, o total de $54,96 \%$ foram classificados como pretos ou pardos (melanoderma ou faioderma).

No que tange ao acesso à educação formal pelas pessoas encarceradas no País, $108.630(52,2 \%)$ possuem apenas fundamental completo e $49.963(24,04 \%)$ fundamental incompleto na faixa etária entre 18 e 24 anos, com baixa escolaridade e baixa renda, estando abaixo ou na linha da pobreza, com histórico de reincidência e cuja condenação, em grande parte, foi motivada por crimes contra o patrimônio e tráfico de entorpecentes. Esses dados confirmam a inclusão da população carcerária no perfil da pesquisa citada anteriormente (BNMP, 2018).

Siqueira et al. (2020), constatam que no cenário populacional carcerário brasileiro encontram-se predominantemente indivíduos jovens, pobres, com baixa escolaridade e com histórico de reincidência, cuja condenação, em grande parte, foi motivada por crimes contra o patrimônio e tráfico de entorpecentes.

Informações relativas à escolaridade do Levantamento Nacional de Informações Penitenciárias destacam que o estado de Alagoas apresenta percentual de analfabetos e alfabetizados (sem cursos regulares), três vezes maior que a média nacional. A Bahia possui 52\% com ensino fundamental incompleto, $15 \%$ alfabetizada (sem curso regular) e 10\% da população analfabeta (INFOPEN, 2016).

De acordo com dados levantados no Plano Nacional de Política Criminal e Penitenciária, oito em cada 10 pessoas presas estudaram, no máximo, até o ensino fundamental, enquanto a média nacional de pessoas que não frequentaram o ensino fundamental ou o têm incompleto é de 50\% (BRASIL, 2015).

Ainda de acordo com o conselho que elabora o plano supracitado o sistema carcerário é quase que exclusivamente composto por pessoas de baixa renda, repercutido na baixa escolaridade. Portanto, a concentração da população carcerária por pessoas de baixa renda é uma característica que se perpetua no sistema punitivo brasileiro, sendo influenciado por estereótipos e padrões que favorecem a inclusão de pessoas pobres no sistema carcerário. Consequentemente, é necessário reconhecer a maior vulnerabilidade das pessoas de baixa renda e/ou baixa escolaridade, ao poder punitivo procurando enfrentar as razões que levam a esse quadro.

Foi observado nos dados do Levantamento Nacional de Informações Penitenciarias, em relação à cor/raça/etnia de uma amostra de 493.145 indivíduos, 
que $64 \%$ da população prisional é composta por pessoas negras, sendo que nessa classificação a categoria negra é constituída pela soma das categorias preta e parda. Esse levantamento também relata que na população brasileira acima de 18 anos a parcela negra representou $53 \%$ do total, indicando a sobre representação deste grupo populacional no sistema prisional. Sendo que no estado do Acre, essa população representa 95\%, no Amapá 91\% e na Bahia 89\% (INFOPEN, 2016).

Segundo o Conselho Nacional de Políticas Criminal e Penitenciária, que realizou estudo em 2015 relacionando à realidade racial da comunidade carcerária em todo o território nacional, $70 \%$ dos indivíduos privados de liberdade são negros, tendo em vista que $51 \%$ da população geral do País é negra. Esse estudo relata que dois em cada três presos são negros, pois a porcentagem de pessoas negras no sistema prisional é de $67 \%$, na população brasileira em geral, a proporção é de $51 \%$. Diante desse cenário observa-se que a faixa populacional historicamente menos favorecida é um eixo estruturante da política criminal brasileira, sendo uma prática que perdura e coloca a população negra em situação de desvantagem até os dias atuais. Portanto, o combate ao racismo orienta os desafios a serem enfrentados do ponto de vista das pessoas criminalizadas, assim como das metodologias e das práticas adotadas no interior do sistema de justiça criminal (BRASIL, 2015).

O combate às desigualdades sociais existentes no território brasileiro tem sido bastante debatido entre os pesquisadores e formuladores de políticas públicas, a fim elaborar e determinar medidas eficazes para a redução desta realidade. A discussão central acontece sobre a temática cor/raça/etnia, uma vez que este aspecto é relativo às importantes características voltadas à história e desenvolvimento do Brasil. Este fato serve como parâmetro para avaliação da composição do sistema carcerário brasileiro, onde grande parte dos detentos se autodeclaram pardos e pretos (faioderma e melanoderma).

Muito embora os cidadãos que possuem tal característica étnico-racial representem grande parte da população do País, esses ainda estão às margens da discriminação racial, o que impacta diretamente nas dificuldades de inserção no mercado de trabalho, baixo grau de escolaridade, renda e aumento das diferenças sociais. Nos últimos anos houve um aumento significativo no percentual de estudantes pretos e pardos no ensino superior, entretanto as desigualdades étnico raciais ainda são evidentes. Além disso, estudos apontam que negros tem 2,7 vezes mais chances de serem vítimas de homicídios, quando comparados a indivíduos brancos. Tais levantamentos enfatizam e comprovam a necessidade e urgência da criação de politicas públicas eficazes no combate e diminuição das desigualdades sociais (IBGE, 2019a,b).

\section{Índice de Dentes Cariados, Perdidos e Obturados (CPO-D)}

No estudo, foram examinados um total de 7.648 dentes. Desse total, 1.840 $(24,05 \%)$ apresentavam experiência de cárie, 402 (5,26\%) restaurados e 892 $(11,67 \%)$ perdidos (extraídos). A mediana do índice de CPO-D correspondeu a 13,1. Quando analisados os componentes do CPO-D isoladamente, observou-se que o componente "dentes cariados" apresentou o maior índice, representando 1.840 $(24,05 \%)$ (Tabela 2).

Em conformidade com a Organização Mundial de Saúde, o CPO-D de indivíduos adultos pode ser categorizado da seguinte forma: CPO-D< 5,0 (muito baixo); CPO-D de 5,0 a 8,9 (baixo); CPO-D de 9,0 a 13,9 (moderado); CPO-D>13,9 (alto) (WHO, 2013). Segundo esta avaliação e como referido anteriormente, o CPOD médio dos encarcerados foi classificado como moderado (BRASIL, 2012). 
TABELA 2 - Frequência por componentes do índice CPO-D dos encarcerados de um conjunto penal em Lauro de Freitas, BA, 2018.

\begin{tabular}{|l|r|}
\hline CPO-D/Componentes & $\mathbf{n ~ ( \% )}$ \\
\hline Cariado & $1.840(24,05 \%)$ \\
\hline Restaurado & $402(5,26 \%)$ \\
\hline Extraído & $892(11,67 \%)$ \\
\hline Hígido & $4.514(59,02 \%)$ \\
\hline Total & $\mathbf{7 . 6 4 8 ( 1 0 0 , 0 0 \% )}$ \\
\hline
\end{tabular}

Fonte: autores (2018).

O CPO-D médio encontrado neste estudo foi de 13,1 sendo considerado moderado. Diferindo do levantamento realizado por Siqueira et al. (2020) com pessoas privadas de liberdade em uma cadeia pública para jovens e adultos, cujo CPO-D médio dos encarcerados com 35 a 44 anos foi de 7,58 (baixo).

O valor no presente estudo, quando comparado a média observada nos indivíduos adultos avaliados no SB Brasil em 2010, comprova que a população encarcerada apresentou melhores condições de saúde bucal do que a população geral, uma vez que, o CPO-D médio dos indivíduos na faixa etária de 35 a 44 foi de 16,75 (alto), e especificamente para a região Nordeste, essa média foi de 16,62 (alto), e em Salvador o valor foi de 14,26 (alto) (BRASIL, 2012).

Inúmeras dificuldades são encontradas no sistema penitenciário, que interferem de forma direta na assistência odontológica. Dentre tais condições, podese citar a superlotação, estrutura precária, e a falta de recursos para serviços odontológicos que contribuem para precariedade da assistência à saúde bucal e surgimento de várias patologias como a cárie dentária. Além disso, é importante ressaltar que grande parte das patologias bucais são ocasionadas devido à ausências de cuidados adequados com a higiene bucal, além da falta de acesso aos serviços odontológicos anteriormente a prisão, uma vez que no momento da admissão já são notadas condições bucais precárias que acabam sobrecarregando o sistema de saúde odontológico das unidades carcerárias (WALSH et al., 2008, HOWARD, 2019).

Em um estudo feito em uma instituição carcerária no Pará, concluiu-se que grande parte dos participantes possuía uma condição de alto risco de desenvolvimento da doença cárie. Notou-se também que, de acordo com o tempo de permanência dos detentos nos presídios, a ocorrência de dentes cariados e perdidos aumentava. Além disso, uma grande parte dos detentos havia sofrido cerca de um ou dois traumas dentários, além de apresentar um grande grau de edentulismo, evidenciando uma alta demanda de reabilitação bucal por meio da confecção de próteses dentárias (ARAÚJO et al., 2013).

Segundo Siqueira et al. (2020), os serviços odontológicos realizados nas instituições carcerárias, restringem-se apenas à eliminação do foco da dor, por meio de tratamentos mutiladores como exodontias de dentes destruídos por atividade da doença cárie, traumatismos ou outras patologias que estivessem provocando dor ao paciente. Além disso, reforça que também eram realizados tratamentos de infecções dentárias (drenagem de abscesso, intra e extraoral) e pequenas suturas, em região de lábio e face, o que geralmente sugerem lesões provenientes de agressões. Esses autores também destacam que os serviços assistenciais odontológicos no contexto carcerário, geralmente se resumem na exodontia, evidenciando a insuficiência ou inexistência de ações voltadas à prevenção, manutenção e recuperação da 
integridade dentária, a fim de proporcionar melhores condições de saúde bucal para os presidiários.

É importante ressaltar que no presente estudo foram observadas semelhanças e divergências entre a presente pesquisa e demais estudos analisados em função da localização da região em que as instituições carcerárias estão situadas, a realidade demográfica e demais fatores influentes nas condições de saúde da população. Portanto, considera-se a análise do índice de CPO-D uma importante ferramenta capaz de inferir as reais condições de saúde bucal de uma dada população em relação as variáveis socioeconômicas e demográficas.

Um estudo transversal de Cavalcanti et al. (2014) avaliou o uso de serviços odontológicos e cárie dentária entre 127 presidiários brasileiros, no estado da Paraíba. Os dados foram coletados por entrevista e exame clínico. Informações sociodemográficas, uso de serviços odontológicos, morbidade dentária autorreferida, autopercepção e impacto da saúde bucal foram investigados. O valor médio do índice CPO-D neste grupo foi de 19,72. O que chama atenção nestes resultados é a associação estatisticamente significante entre CPO-D com valores de 22 a 32 e insatisfação com a saúde bucal $(p=0,002)$, dificuldade para falar $(p=0,024)$, vergonha de falar $(p=0,004)$ e sorrir $(p<0,001)$. Em relação à utilização de serviços odontológicos, $80 \%$ tiveram sua última consulta com dentista há menos de um ano, com a maioria das visitas ocorrendo na prisão.

Levando-se em consideração que as infecções bucais causam dor, estresse, além de agravos importantes para a saúde sistêmica dos indivíduos, fica evidente a necessidade de controle dessas doenças. $\mathrm{E}$, de forma mais particular, considerando indivíduos mantidos em cárcere, a diminuição do nível de estresse impacta de forma muito positiva no controle da violência no contexto dos presídios (MINAYO; RIBEIRO, 2016).

Extrapolando a análise da condição individual de saúde, estudos longitudinais sobre a condição bucal da população carcerária podem refletir a forma como são conduzidos os serviços de assistência à saúde dentro das unidades penitenciárias. Dados desse tipo de estudo podem auxiliar a melhoria da implementação da assistência odontológica, uma vez que contribuem para a identificação dos fatores de risco inerentes à incidência e prevalência de doenças bucais.

\section{CONCLUSÕES}

O perfil dos presidiários avaliados foi composto por indivíduos do sexo masculino, faioderma, idade média de 42,5 anos, e que estudaram até o ensino fundamental. Em relação à condição bucal, 24,05\% da população carcerária avaliada apresentaram experiência de cárie, 5,25\% possuíam dentes restaurados e $11,66 \%$ foram perdidos/extraídos. A mediana do índice de CPO-D correspondeu a 13,1 sendo este considerado uma condição moderada $(9,0$ a 13,9$)$ pela Organização Mundial de Saúde (OMS).

Assim, é possível inferir que os detentos avaliados apresentam uma condição de saúde bucal moderada. Sendo as mais altas porcentagens de dentes cariados e perdidos, havendo baixa porcentagem de dentes restaurados, evidenciam a falta de acesso e pouca adesão aos tratamentos odontológicos nesta população.

Há necessidade de mais estudos de forma ampliada, de modo que permitam visualizar as reais condições de saúde bucal dos presidiários no intuito de auxiliar o planejamento dos gestores no que se refere a ações de saúde bucal nas unidades de saúde presente nos sistemas prisionais, viabilizando a oferta de uma atenção odontológico integral e eficiente neste ambiente. 


\section{AGRADECIMENTOS}

Aos funcionários da instituição prisional, em especial ao diretor pela autorização da coleta de dados, ao cirurgião-dentista responsável pelo suporte e disponibilização dos prontuários. Ao Fernando Chagas pelo auxilio nas coletas, a FAMAM pela bolsa de estudos de mestrado concedida ao terceiro autor.

\section{REFERENCIAS}

ALVES, J. P.; BRAZIL, J. M.; NERY, A. A.; VILELA, A. B. A.; MARTINS FILHO, I. E. Perfil epidemiológico de pessoas privadas de liberdade. Revista de Enfermagem da UFPE on line, p. 4036-4044, 2017. Disponível em: http://dx.doi.org /10.5205/reuol.10712-95194-3-SM.1110sup201705

ARAÚJO, R. J.; REIS, A. C. A.; ALMEIDA, A. P. G. Análise de cárie e edentulismo da população carcerária do hospital de custódia e tratamento psiquiátrico do estado do Pará. Expediente Editorial, 66035, 29, 2013. Disponível em: https://www.researchgate.net/profile/Lidiane_Coelho/publication/263470160_Avaliac ao_clinica_da_relacao_entre_disturbios_oclusais_e_a_ocorrencia_da_recessao_gen gival//links/0a85e53cac5fa96328000000.pdf\#page $=29$

BNMP - Banco Nacional de Monitoramento de Prisões - 2.0: Cadastro Nacional de Presos, Conselho Nacional de Justiça, Brasília, agosto de 2018. Disponivel em https://www.cnj.jus.br/sistema-carcerario/cadastro-nacional-de-presos-bnmp-2-0/

BRASIL. Ministério da Saúde - Conselho Nacional de Saúde, Resolução no 466. 2012 Disponível em: http://bvsms.saude.gov.br/bvs/saudelegis/cns/2013/res0466_12_12_2012.html\& t; acesso: 01 de Junho de 2018.

BRASIL. Ministério da Saúde. Secretaria de Atenção em Saúde. Departamento de Ações Programáticas Estratégicas. Legislação da saúde no sistema

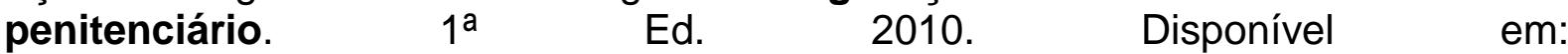
http://bvsms.saude.gov.br/bvs/publicacoes/legislacao_saude_sistema_penitenciario. pdf. Acesso em: 23 nov. 2017.

BRASIL. Plano Nacional de Política Criminal e Penitenciária. Conselho Nacional de Política Crimanal e Penintenciária, 2015. Disponível em: http://www.justica.gov.br/seus-direitos/politica-penal/cnpcp-1/imagens- cnpcp/plano nacional-de-politica-criminal-e-penitenciaria-2015.pdf\&gt; Acesso em 19 de Maio 2018.

CAVALCANTI, A. L., RODRIGUES, I. S., ARAUJO, A., DE MELO SILVEIRA, I. T., DE OLIVEIRA, T. B. S.; et al. Dental caries experience and use of dental services among Brazilian prisoners. International journal of environmental research and public health, v. 11, n. 12, p. 12118-12128, 2014. Disponível em:

http://dx.doi.org/10.3390/ijerph111212118

FREITAS, R. S.; ZERMIANI, T. C.; NIEVOLA, M. T. S.; NASSER, J. N.; DITTERICH, R. G. Política Nacional de Atenção Integral à Saúde das Pessoas Privadas de Liberdade no Sistema Prisional: uma análise do seu processo de formulação e 
implantação. Revista de Políticas Públicas, v. 20, n. 1, p. 171-184, 2016. Disponível em: http://dx.doi.org/10.18764/2178-2865.v20n1p171-184

HOWARD, P. Providing dental care in prisons. Dental Update - Special Care Dentistry, v. 46, n. 5, 2019. Disponível em: https://doi.org/10.12968/denu.2019.46.5.428

IBGE. Instituto Nacional de Geografia e Estatística. Desigualdades sociais por cor ou raça no Brasil. Estudos e Pesquisas, Informação Demográfica e Socioeconômica, n. 41. 2019a.

IBGE. Instituto Nacional de Geografia e Estatística. Pretos ou pardos estão mais escolarizados, mas desigualdade em relação aos brancos permanece. $2019 \mathrm{~b}$. Disponível em: <https://agenciadenoticias.ibge.gov.br/agencia-sala-deimprensa/2013-agencia-de-noticias/releases/25989-pretos-ou-pardos-estao-maisescolarizados-mas-desigualdade-em-relacao-aos-brancos-permanece>.

INFOPEN - Levantamento Nacional de Informações Penitenciárias. Brasília: Ministério da Justiça e Segurança Pública. Departamento Penitenciário Nacional, 2016. Disponível em: http://dados.mj.gov.br/dataset/infopen-levantamento-nacionalde-informacoes-penitenciarias. Acesso em: 23 nov. 2016.

INFOPEN - Levantamento Nacional de Informações Penitenciárias. Brasília: Ministério da Justiça e Segurança Pública. Departamento Penitenciário Nacional, 2020. Disponível em: http://dados.mj.gov.br/dataset/infopen-levantamento-nacionalde-informacoes-penitenciarias. Acesso em: 23 ago. 2020.

MINAYO, M. C. D. S.; RIBEIRO, A. P. Condições de saúde dos presos do estado do Rio de Janeiro, Brasil. Ciência \& Saúde Coletiva, v. 21, p. 2031-2040, 2016. Disponível em: http://dx.doi.org/10.1590/1413-81232015217.08552016.

SILVA, A. F.; GOMES, J. R.; OLIVEIRA, K. V. S.; SILVEIRA, V. B. C. A visibilidade dos invisíveis: uma análise sobre o acesso à saúde da pessoa privada de liberdade e a intervenção do serviço social no âmbito da defensoria pública do estado de São Paulo- Regional Se Presidente Prudente. Intertem@s Social, v. 11, n. 11, 2016. Disponível

em:

http://intertemas.toledoprudente.edu.br/index.php/Social/article/view/5950

SILVA, E. L. A realidade do sistema penitenciário brasileiro e o princípio da dignidade da pessoa humana. DireitoNet, p. 1-6, 2017. Disponível em: https://www.direitonet.com.br/artigos/exibir/7926/A-realidade-do-sistemapenitenciario-brasileiro-e-o-principio-da-dignidade-da-pessoa-humana

SIQUEIRA, M. R., BOAS, M. C. R. V.; ABUD, J. I. F.; DE ARAÚJO, R. J. G.; DOS REIS, A. C. A. Saúde bucal da população carcerária: levantamento epidemiológico. Journal of Research in Dentistry, v. 7, n. 6, p. 91-106, 2020. Disponível em: http://dx.doi.org/10.19177/jrd.v7e6201991-106

SOARES FILHO, M. M.; BUENO, P. M. M. G. Demografia, vulnerabilidades e direito à saúde da população prisional brasileira. Ciência \& Saúde Coletiva, v. 21, p. 1999- 
2010, 2016. Disponível em: https://doi.org/10.1590/1413-81232015217.24102015

TETZNER, E.; NASCIMENTO, S.; CARVALHO, R.; TONINI, K. Odontologia no sistema penal. Revista da Faculdade de Odontologia-UPF, v. 17, n. 3, 2012. Disponível em: http://files.bvs.br/upload/S/1413-4012/2012/v17n3/a3724.pdf. Acesso em: 23 nov. 2017.

WALSH, T.; TICKLE, M.; MILSOM, K.; BUCHANAN, K.; ZOITOPOULOS, L. British Dental Journal, v. 204, n. 12, p. 683-689, 2008. Disponível em: http://dx.doi.org/10.1038 / sj.bdj.2008.525

WHO. World Health Organization. Oral health surverys: basic methods. 5th ed. Geneva: The organization; 2013. em:https://www.who.int/oral_health/publications/9789241548649/en/ 